\title{
54. Possible Evidence for the Karyotype Evolution of the Indian Spiny Mouse due to Tandem Fusion of the House Mouse Chromosomes*)
}

\author{
By Tosihide H. YosidA \\ National Institute of Genetics, Misima \\ (Communicated by Sajiro Makino, M. J. A., June 12, 1979)
}

It has been reported that the karyotype of the Indian spiny mouse, Mus platythrix, is characterized by $2 n=26$ in which all chromosome pairs are acrocentrics (Satya Prakash and Aswathanarayana 1972; Tsuchiya and Yosida 1972; Dhanda et al. 1973; Yosida 1978). On the basis of the measurement of chromosome length as well as of the Gband analysis between the spiny mouse and the house mouse (Mus musculus), the assumption became possible that several chromosome pairs of the spiny mouse have resulted from the tandem fusion of acrocentrics of the house mouse. In this paper is presented a preliminary note on the development of the karyotype of the Indian spiny mouse due to the tandem fusion.

Material and method. The Indian spiny mice (Mus platythrix) for the present study were collected in Mysore, India in 1972, and then they have been bred in our laboratory for the last six years. The house mice (M. musculus) from the B10A strain of our laboratory stock were served as material.

Chromosomes were studied based on bone marrow as well as on lung cultures. The trypsin G-banding, $\mathrm{Ba}(\mathrm{OH})_{2}$ C-banding (Yosida and Sagai 1975), and Goodpasture and Bloom's Ag-AS technique (1975) for the nucleolar organizer were applied to chromosome analyses.

Results. 1) Karyotype of the Indian spiny mouse: The chromosome number of the Indian spiny mouse was $2 n=26$, all of which were acrocentrics of varying sizes (Fig. 1). All chromosome pairs of the animal were rather easily identified by the G-banding technique (Fig. 2). C-bands of the spiny mouse were generally small and obscure in outline. Interstitial C-bands were observed in some autosome pairs (Fig. 1B). This species was noted by having a typical nucleolar organizer (Ag-NOR) in the following three chromosome pairs: nos. 5, 8 and 12 (Fig. 1C).

*) Contribution No.1264 from the National Institute of Genetics, Japan. Supported by a grant-in-aid for the scientific research from the Ministry of Education, Science and Culture (Nos. 339025, 301001). 


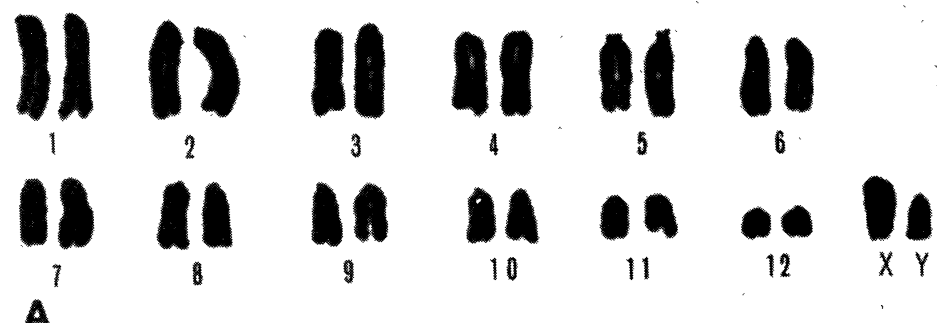

A

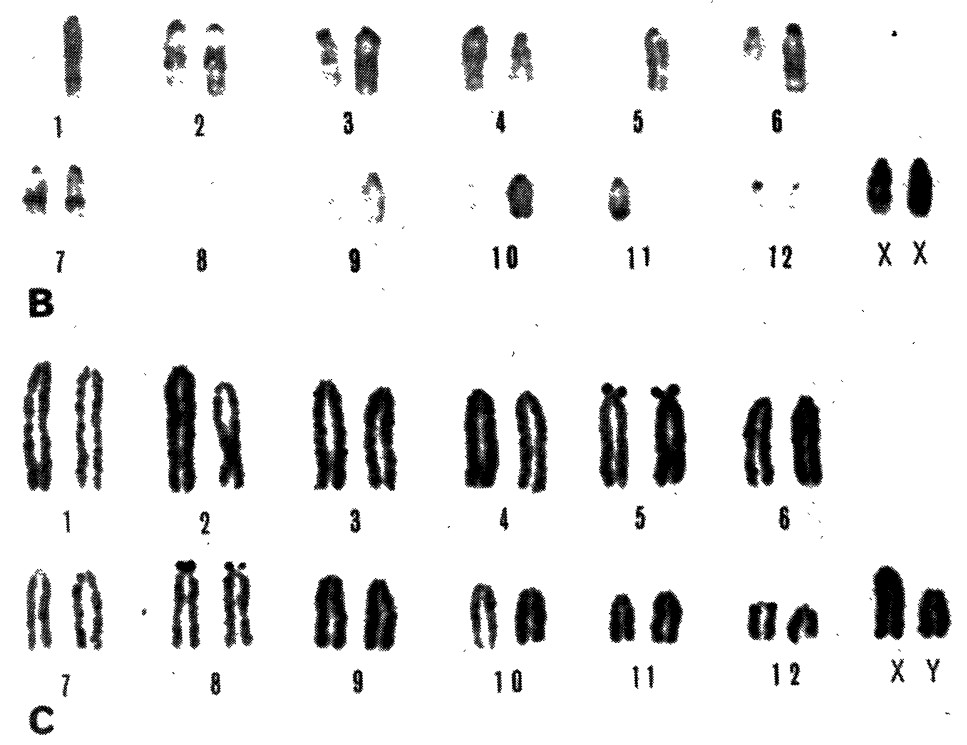

Fig. 1. Karyotypes of the Indian spiny mouse. A: Conventional staining (令). B: C-band staining (ㅇ). C: Ag-NOR's staining (合). The NOR regions are demonstrated in pair nos. 5, 8 and 12 .

2) Comparison of chromosomes between the spiny mouse and the house mouse: The chromosome numbers were different between the Indian spiny mouse $(2 n=26)$ and the house mouse $(2 n=40)$, although their complement was consisted of acrocentric chromosomes. After the comparison of the G-bands between the spiny mouse and the house mouse has led to the possible conclusion was induced that several chromosome pairs of the spiny mouse could be derived due to the tandem fusion of two acrocentric pairs occurring in the house mouse (Fig. 2). The banding pattern in the proximal half of the longest no. 1 chromosome found in the spiny mouse was similar in structure 
to that of pair no. 8 of the house mouse, and further the distal half of the former was identical with pair no. 1 of the house mouse. Pair no. 2 of the spiny mouse chromosomes seemed to be formed by the tandem fusion between pair nos. 5 and 6 of the house mouse. By a similar way, pair nos. 3, 4, 5 and 6 of the spiny mouse chromosomes seemed to be derived from the tandem fusion between pair nos. 12 and 4, between nos. 10 and 13, between nos. 16 and 7 , and between nos. 14 and 17 of the house mouse chromosomes. Pair no. 7 of the spiny mouse could be produced by fusion of the proximal half of pair no. 3 and pair no. 11 of the house mouse. Due to the tandem fusion of the distal half of pair no. 3 and pair no. 12 of the house mouse chromosomes, pair no. 8 of the spiny mouse seemed to be resulted. Pair nos. 9, 10, 11 and 12 of the spiny mouse seemed to correspond to pairs 2, 9, 18 and 19 of the house mouse respectively, although there was in each a slight difference in the chromosome length between these two forms.

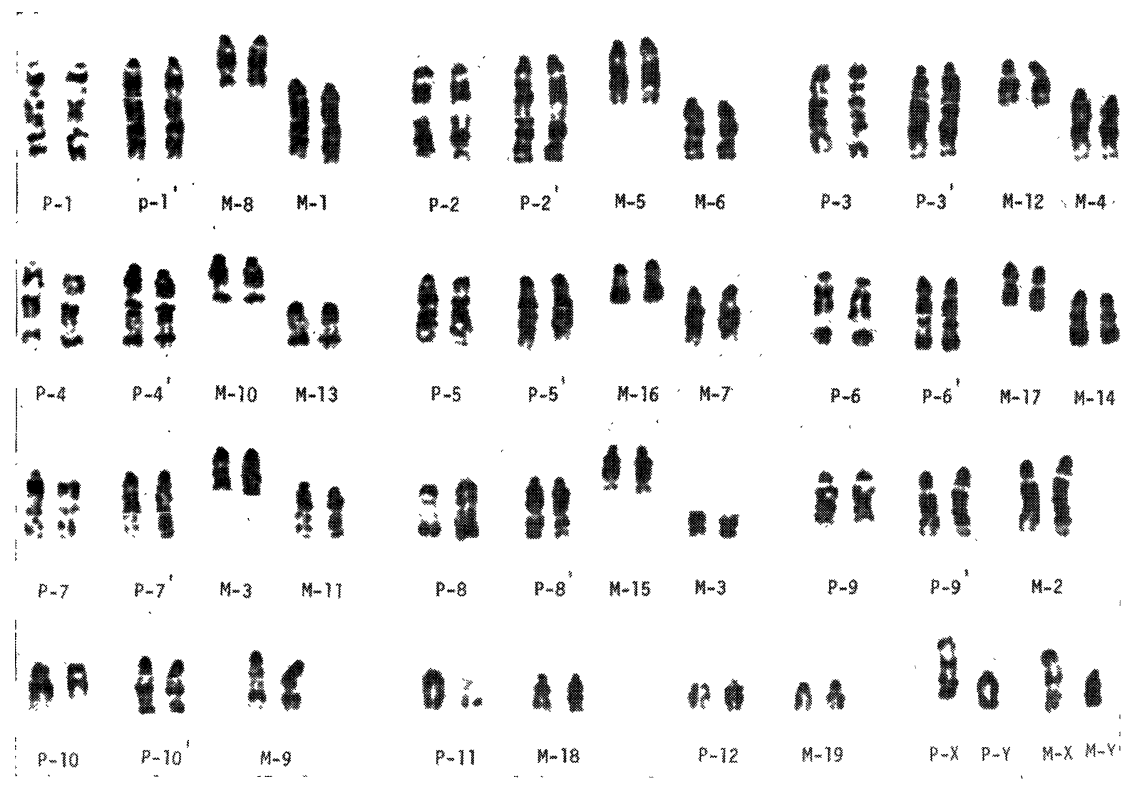

Fig. 2. Comparison of G-band chromosomes between the spiny mouse (Mus platythrix) and the house mouse (M. musculus). P-1, P-2, ... denote the chromosome numbers of platythrix, and M-1, M-2, .., those of musculus. P-1', P-2',... show the chromosome numbers of platythrix, which are artificially constructed from musculus chromosomes.

The total average length of chromosomes (a haploid autosome set $+\mathrm{XY}$ ) obtained on the basis of 30 cels was $82.6 \mu$ in the house mouse, while in the spiny mouse it was $72.0 \mu$. In the total chromosome 
length, the spiny mouse was about $10.6 \mu$ shorter than the house mouse. Thus it appeared that about 12.8 per cent of centromere and telomere regions of the house mouse chromosomes seems to be lost for production of the spiny mouse chromosomes by the tandem fusion.

Remarks. Robertsonian rearrangements or the fusion-fission mechanism of chromosomes has been known as a common phenomenon for the karyotype evolution in mammals. Example of the Robertsonian fusion has been found in the tobacco mouse (Mus poschiavinus), the black rat (Rattus rattus) and some others, while the Robertsonian fission has recently been demonstrated in Rattus rattus (Yosida et al. 1979). The number of chromosomes of the tobacco mouse was 26 $(n=13)$, being the same as that of the spiny mouse. According to Gropp et al. (1970) the former species had 7 large metacentric pairs which were derived from the Robertsonian fusion of 14 acrocentrics of the house mouse. The diploid complement of the Indian spiny mouse, however, was consisted of all of acrocentric members. Comparative banding analyses of the chromosomes between the spiny mouse and the house mouse have made it possible to see that certain chromosome pairs of the spiny mouse could be resulted from the tandem fusion of the house mouse chromosomes.

The chromosomes of the Indian muntjac, Muntiacus muntjak, have provided a fair example of the tandem fusion in their origin. This species is characterized by having a very low chromosome number as $2 n=6$. This chromosome complement has probably been derived by sequential tandem fusion of some chromosome pairs in the other Muntiacus species with $2 n=46$ (Wurster and Benirshke 1970). For the karyotype evolution due to the tandem fusion two processes are to be considered. One is that two chromosome pairs are involved in each tandem fusion as seen in the spiny mouse, while the other corresponds to the case of sequential tandem fusion as seen in the muntjac. In any case a number of centromeres are lost from the original karyotype as a result of the tandem fusion. The number of centromeres to be lost from the original karyotype should be shown by a formula of $\frac{n}{2}$ in the latter case (the muntjac type), and in the former case (the spiny mouse type) by a formula of $n-1$ (" $n$ " being the number of chromosomes involved in the tandem fusion).

There is another explanation for the tandem fusion hypothesis that the karyotypes with a lower chromosome number are an original type, and that these with a higher chromosome number are derived from the former by tandem fission of chromosomes. If the tandem fission hypothesis is accepted, the new formation of many centromeres and telomeres is needed for the production of a new karyotype. In 
the present situation, we will present a tentative explanation on the origin of the Indian spiny mouse karyotype due to the tandem fusion of acrocentrics of the house mouse karyotype.

Summary. Chromosomes of the Indian spiny mouse (Mus platythrix) are studied by the conventional and banding techniques with special reference to their origin. This species has $2 n, 26$ comprizing 12 acrocentric autosome pairs and acrocentric $\mathrm{X}$ and $\mathrm{Y}$ chromosomes. The comparison of G- and C-banded chromosomes and the measurement of the chromosome length between the spiny mouse and the house mouse (M. musculus), have made it possible to suggest that 8 autosome pairs (nos. 1 to 8 ) of the former species have been derived from the tandem fusion of two acrocentric pairs of the latter species. In this case, about 12.8 per cent of the centromere and telomere regions of chromosomes seems to be lost from the original karyotype to construct the new formation.

Acknowledgements. The author is indebted to Mr. Tooru Inoue for his skillful technical assistance to the present work. The author also is very grateful to Emeritus Professor Sajiro Makino, M. J. A., for revision of the manuscript with keen interests and continued encouragement.

\section{References}

Dhanda, V., Mishra, A. C., Bhat, K. M., and Wagh, U. V.: The Nucleus, 16, 56-59 (1973).

Goodpasture, C., and Bloom, E.: Chromosoma (Berl.), 53, 37-50 (1975).

Gropp, A., Tettenborn, U., and Lehmann, E. von: Cytogenetics, 9, 9-23 (1970).

Satya Parakash, K. L., and Aswathanarayana, N. V.: Mamm. Chrom. Newsl., 13, $120(1972)$.

Tsuchiya, K., and Yosida, T. H.: Ann. Rep. Nat. Inst. Genet. Japan, 22, 51-52 (1972).

Wurster, D. H., and Benirschke, K.: Science, 168, 1364-1366 (1970).

Yosida, T. H.: Ann. Rep. Nat. Inst. Genet. Japan, 28, 66-67 (1978).

Yosida, T. H., Kato, H., Tsuchiya, K., Moriwaki, K., Ochiai, Y., and Monty, J.: Proc. Japan Acad., 55B, 120-125 (1979).

Yosida, T. H., and Sagai, T.: Chromosoma (Berl.), 50, 283-300 (1975). 